$\mathrm{Cu}^{2+}$ ions. It is this change in the magnetic background associated with hole doping that leads to pairing.

Over the past 30 years or so, researchers have looked for superconductivity in other compounds that have planes containing spin- $1 / 2$ ions. Examples of such compounds are $\mathrm{LaNiO}_{2}$ and $\mathrm{NdNiO}_{2}$, which comprise alternating planes of lanthanum or neodymium and $\mathrm{NiO}_{2} . \mathrm{Ni}^{1+}$ ions in these materials could have the same role in inducing superconductivity as do $\mathrm{Cu}^{2+}$ ions in $\mathrm{La}_{1.85} \mathrm{Ba}_{0.15} \mathrm{CuO}_{4}$. Several groups have prepared $\mathrm{LaNiO}_{2}$ and $\mathrm{NdNiO}_{2}$ in both powder and thin-film form (see, for example, refs 6-8). However, no superconductivity (but also no sign of magnetic order) has been found.

Enter Li and colleagues. The authors grew a thin film of $\mathrm{NdNiO}_{2}$ and then hole-doped this film by replacing some $\mathrm{Nd}^{3+}$ ions with $\mathrm{Sr}^{2+}$ ions. They found that the resulting material, $\mathrm{Nd}_{0.8} \mathrm{Sr}_{0.2} \mathrm{NiO}_{2}$, superconducts at temperatures of up to $15 \mathrm{~K}$. After some 30 years of trying, scientists have finally found a non-cuprate compound that has a cuprate-like structure and that exhibits superconductivity at surprisingly high temperatures. But, unlike in the cuprates, there is no sign of magnetic order in $\mathrm{NdNiO}_{2}$ down to a temperature of $1.7 \mathrm{~K}$. The authors' discovery might therefore indicate that magnetism is not exclusively responsible for cuprate superconductivity.

However, this conclusion is based on the assumption that the cuprates and hole-doped $\mathrm{NdNiO}_{2}$ have similar electronic structures. There are three reasons why this assumption might not be valid. First, in the cuprates, the holes reside mainly in the $2 p$ electron orbitals of oxygen atoms. The spins of these holes couple antiferromagnetically to the spins of neighbouring $\mathrm{Cu}^{2+}$ ions, producing a net spin of 0 . By contrast, in hole-doped $\mathrm{NdNiO}_{2}$, the holes reside mostly in $\mathrm{Ni}^{1+}$ ions and result in $\mathrm{Ni}^{2+}$ ions that, in conventional oxides, have a spin of 1 (ref. 9). But perhaps the situation here is different from that of conventional oxides. $\mathrm{X}$-ray spectroscopy could determine whether this is the case, if good enough samples are available.

Second, the antiferromagnetic coupling between spins might be substantially stronger in the cuprates than in $\mathrm{NdNiO}_{2}$. This difference would be consistent with the absence of magnetic order in $\mathrm{NdNiO}_{2}$. And third, a theoretical study ${ }^{10}$ suggests that $5 d$ electron orbitals of lanthanum atoms in $\mathrm{LaNiO}_{2}$ and of neodymium atoms in $\mathrm{NdNiO}_{2}$ are involved in electrical transport. If confirmed, this result could change the picture completely. In particular, local spins would be affected by being coupled to delocalized conducting electrons, as in compounds called Kondo systems ${ }^{11}$. Such systems exhibit a minimum in a plot of resistivity against temperature, which is observed by Li et al. for $\mathrm{NdNiO}_{2}$.

There are therefore many issues to address before it can be concluded that the electronic structures of the cuprates and of hole-doped $\mathrm{NdNiO}_{2}$ are similar. Future work should check that the nickel ions in $\mathrm{NdNiO}_{2}$ are $\mathrm{Ni}^{1+}$ ions, determine the local symmetry and spin of the hole-doped states and explore how the temperature at which the material becomes superconducting varies with hole doping. The chemical composition of the material also needs to be verified, because unwanted hydrides or hydroxides might have formed. Nevertheless, Li and colleagues' work could become a game changer for our understanding of superconductivity in cuprates and cuprate-like systems, perhaps leading to new high-temperature superconductors. -

George A. Sawatzky is at the Stewart Blusson Quantum Matter Institute and in the Department of Physics and Astronomy,
University of British Columbia, Vancouver, British Columbia V6T 1Z1, Canada.

e-mail:sawatzky@physics.ubc.ca

1. Bednorz, J. G. \& Müller, K. A. Z. Phys. B 64, 189-193 (1986).

2. Schilling A., Cantoni, M., Guo, J. D. \& Ott, H. R Nature 363, 56-58 (1993).

3. Li, D. et al. Nature 572, 624-627 (2019).

4. Bardeen, J., Cooper, L. N. \& Schrieffer, J. R. Phys. Rev. 106, 162-164 (1957)

5. Mott, N. F. \& Peierls, R. Proc. Phys. Soc. 49, 72-73 (1937).

6. Crespin, M., Levitz, P. \& Gatineau, L. J. Chem. Soc. Faraday Trans. 2 79, 1181-1194 (1983).

7. Hayward, M. A., Green, M. A., Rosseinsky, M. J. \& Sloan, J. J. Am. Chem. Soc. 121, 8843-8854 (1999).

8. Hayward, M. A. \& Rosseinsky, M. J. Solid State Sci. 5, 839-850 (2003).

9. Zaanen, J., Sawatzky, G. A. \& Allen, J. W. Phys. Res. Lett. 55, 418-421 (1985).

10.Lee, K.-W. \& Pickett, W. E. Phys. Rev. B 70, 165109 (2004).

11.Kondo, J. Prog. Theor. Phys. 32, 37-49 (1964).

\title{
What makes flatworms go to pieces
}

Flatworms called planarians can break off fragments of themselves that regenerate to form new, complete worms. The molecular cues that regulate the frequency of such fission events have been revealed. SEE LETTER P.655

\section{THOMAS W. HOLSTEIN}

$\mathrm{U}$ nderstanding how tissues and organs can regenerate requires an appreciation of the mechanisms and factors that organize cells and tissues, both in space and through time. Planarian flatworms are a widely used model for studying such pattern formation because pieces of these animals that are cut off can regrow missing body parts and form complete worms. Planarians also have a self-scission behaviour called fission - they stretch and contract their tail tissue, which leads to detachment of parts of their posterior body that then grow into clones. Whether or not fission occurs depends on the size of the parent worm, but the underlying molecular and cellular processes have not been well understood. On page 655, Arnold et al. ${ }^{1}$ establish a method to reliably induce fission in the planarian Schmidtea mediterranea, and show that cell-signalling pathways involving the proteins Wnt and transforming growth factor- $\beta$ (TGF- $\beta$ ) are key regulators of this process.

Wnt signalling has a decisive role in development and cell differentiation and is involved in many diseases ${ }^{2}$. The Wnt proteins are highly diverse, are found only in animals and are usually attached to a lipid chain and secreted by cells. They bind to receptor proteins of different families to activate various downstream cell-signalling cascades that regulate the levels of cytoplasmic factors - molecules that control gene expression and, thus, cell function ${ }^{2,3}$. Although our knowledge of the influence of Wnt signalling on tissue-pattern formation has advanced greatly in the past few years, how such patterning might be linked to specific tissue functions is still unknown.

Previous studies ${ }^{4-6}$ in planarians have characterized a molecular framework in which self-organized gradients of Wnt proteins regulate patterning along the length of the animal (that is, along the anterior-posterior axis), and in which a gradient of TGF- $\beta$ regulates patterning from its topside to its underside (along the dorsal-ventral axis). It has been suggested ${ }^{7}$ that planarian fission is regulated by gradients in metabolic activity, molecular positional cues or neurohormone molecules along the anterior-posterior body axis. One study indicated that fission might be inhibited by the front part of the nervous system ${ }^{7}$, and another examined the biomechanical forces and tissue properties that enable it to occur ${ }^{8}$.

Unlike regeneration, which can be induced experimentally by cutting planarian worms into pieces, fission has been difficult to induce reliably, limiting studies on this process. However, Arnold et al. found that transferring worms to cultures in which food was limited and water was stagnant induced fissioning in worms longer than about 4 or 5 millimetres 


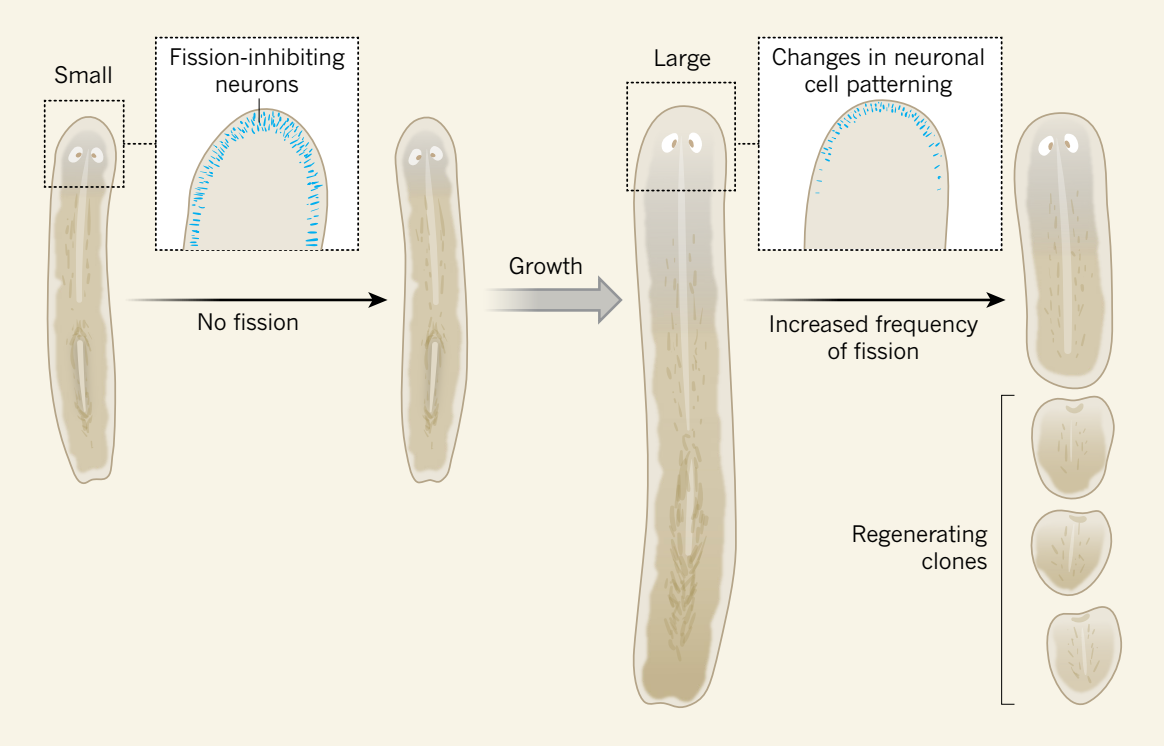

Figure 1 | Size-dependent fission behaviour in planarian flatworms. Planarian flatworms can reproduce through a process called fission. In this process, a worm breaks off a portion of tissue from the back end of its body, and this portion regenerates to form a complete worm. Arnold et al. ${ }^{1}$ examined the molecular and cellular underpinnings of this fission process. They found that the frequency of fission events correlated with the size of the parent animal. Experimental disruptions of the expression of certain proteins involved in the Wnt signalling pathway (not shown), which controls tissue patterning along the length of planarians ${ }^{4,5}$, did not affect the positioning of fission planes along the body, but did increase or reduce the frequency of fission events. The authors showed that Wnt signalling regulates the fine-scale patterning of a population of neuronal cells at the front of the worm (boxes) that inhibit fission behaviour, and showed that the patterning of these neurons changes with animal size.

(Fig. 1). By analysing image recordings, the researchers discovered that fission events take about 30 minutes and result in fragments that are about $1 \mathrm{~mm}$ long, and that the frequency of fission events correlates with the size of the parent. Arnold et al. also found that, when they applied pressure to a cover glass placed on top of a worm in normal culture, the worm would break apart into multiple, regularly spaced fragments along its entire anterior-posterior axis. This suggests that, in adult worms, there are pre-established fission planes that scale in number with the animal's size, and that a hidden, segmented structure underlies this size control.

Using both the starvation and compression methods to induce fission, the authors tested which molecular cues are required to induce size-dependent fissioning. They carried out a screen in which they used different RNA molecules to selectively inhibit the expression of various proteins involved in patterning, including those in the Wnt and TGF- $\beta$ cell-signalling pathways ${ }^{4,6,9,10}$. These targeted disruptions affected fission frequency; for example, blocking the expression of APC, a protein that suppresses the Wnt signalling pathway, roughly doubled the frequency of sequential fission attempts in which the animals showed their characteristic stretching behaviour. However, interfering with these signalling pathways did not affect the positioning of fission planes along the body axis. Thus, Wnt and TGF- $\beta$ signalling seem to regulate fission behaviour independently of their function in axial patterning. cut into pieces. In both cases, populations of stem cells called neoblasts cluster to form a mass called a blastema at the wound site in the tissue fragment, which in turn can regenerate different organs and tissues ${ }^{14}$. But how the information concerning the position of the cut or fission plane is transmitted to neoblasts is not clear.

Asexual reproduction through fission is a major strategy for increasing population size, not only in planarians, but also in other wormlike creatures (including acoels ${ }^{15}$ and other acoelomorph flatworms ${ }^{16}$, and annelids ${ }^{17}$ ) in which fission occurs at the posterior end of the animal. Sea anemones can also propagate asexually through fission ${ }^{18}$, and budding - a fission-related strategy for asexual reproduction - has been well characterized in the freshwater animal $\mathrm{Hydra}^{19}$ and is strongly related to regeneration ${ }^{20}$.

Detailed investigation of fission and budding in different model organisms will be important because, in these processes, pattern formation is induced without injury, and therefore might be different from regeneration after injury. If the processes that enable regeneration in planarians after fission and after cutting are indeed the same, future research should determine the mechanisms that compensate for the lack of an injury signal in fissioning tissue. Such research will be crucial for understanding how injury and patterning signals converge to initiate the regeneration process.

A previous gene-expression analysis ${ }^{11}$ revealed that genes encoding proteins involved in Wnt and TGF- $\beta$ signalling are coexpressed with genes expressed by cells in the central nervous system (CNS). In Arnold and colleagues' study, removing the front part of the worm that contained the cephalic ganglia (two clusters of neurons that together comprise the planarian brain) delayed the onset of fission behaviour. The authors saw a similar effect in worms in which the expression of a neuronal transcription-factor protein that was previously shown to be required for CNS patterning ${ }^{12}$ was suppressed.

Arnold et al. found that a set of neuronal cells that are sensitive to mechanical stimuli act downstream of Wnt and TGF- $\beta$ signalling to inhibit fission behaviour. The authors demonstrated that Wnt and TGF- $\beta$ signalling together regulate the patterning of these and other specific populations of neurons (Fig. 1). It will be exciting to examine how these key regulators of axial patterning control the fine patterning of the planarian nervous system ${ }^{13}$ - one of the big questions about the patterning of different types of cell is how these signalling pathways are integrated by progenitor cells to induce the generation of specific neuronal cell types.

Although Arnold et al. focused their analysis on the induction of fission, even less is known about how the released tissue fragments form complete animals. For example, it is unclear whether these worms regenerate after fission in the same way that they regrow after being
Thomas W. Holstein is in the Department of Molecular Evolution and Genomics, Centre for Organismal Studies, Heidelberg University, 69120 Heidelberg, Germany.

e-mail:thomas.holstein@cos.uni-heidelberg.de

1. Arnold, C. P., Benham-Pyle, B. W., Lange, J. J., Wood, C. J. \& Sánchez Alvarado, A. Nature $\mathbf{5 7 2}$ 655-659 (2019).

2. Steinhart, Z. \& Angers, S. Development $\mathbf{1 4 5}$, dev146589 (2018)

3. Wiese, K. E., Nusse, R. \& van Amerongen, R. Development 145, dev165902 (2018).

4. Gurley, K. A., Rink, J. C. \& Sánchez Alvarado, A. Science 319, 323-327 (2008)

5. Niehrs, C. Development 137, 845-857 (2010).

6. Stuckemann, T. et al. Dev. Cell 40, 248-263 (2017).

7. Best, J. B., Goodman, A. B. \& Pigon, A. Science 164 565-566 (1969).

8. Malinowski, P. T. et al. Proc. Natl Acad. Sci. USA 114, 10888-10893 (2017).

9. Petersen, C. P. \& Reddien, P. W. Cell 139, 1056-1068 (2009)

10.Molina, M. D., Salo, E. \& Cebria, F. Dev. Biol. 311, 79-94 (2007).

11.Collins, J. J. Ill et al. PLoS Biol. 8, e1000509 (2010).

12.Cowles, M. W., Omuro, K. C., Stanley, B. N., Quintanilla, C. G. \& Zayas, R. M. PLoS Genet. 10, e1004746 (2014).

13. Kobayashi, C., Saito, Y., Ogawa, K. \& Agata, K. Dev. Biol. 306, 714-724 (2007).

14. Reddien, P. W. Cell 175, 327-345 (2018).

15.Sikes, J. M. \& Bely, A. E. Dev. Biol. 338, 86-97 (2010).

16.Cannon, J. T. et al. Nature 530, 89-93 (2016)

17.Zattara, E. E. \& Bely, A. E. Evol. Dev. 13, 80-95 (2011)

18. Burton, P. M. \& Finnerty, J. R. Dev. Genes Evol. 219 , 79-87 (2009).

19.Chapman, J. A. et al. Nature 464, 592-596 (2010).

20.Petersen, H. O. et al. Mol. Biol. Evol. 32, 1928-1947 (2015).

This article was published online on 14 August 2019. 